TAIWANESE JOURNAL OF MATHEMATICS

Vol. 10, No. 1, pp. 181-185, January 2006

This paper is available online at http://www.math.nthu.edu.tw/tjm/

\title{
RETRIEVE A SPERNER MAP FROM A SPERNER MATROID
}

\author{
Shyh-Nan Lee and Mau-Hsiang Shih
}

\begin{abstract}
A retrievel of a Sperner map from a Sperner matroid is illustrated. As an application, a new proof of a completion theorem of Lovász's matroid version of Sperner's lemma is given.
\end{abstract}

\section{INTRODUCTION}

The purpose of this note is to give a simple procedure which allows us to retrieve a Sperner map from a Sperner matroid.

\section{Retrieving a Sperner Map on the Basis of a Sperner Matroid}

Let $K$ be a triangulation of a $d$-simplex $a_{0} a_{1} \ldots a_{d}$ in a Euclidean space, and $V(K)$ the vertex set of $K$. A map $\varphi: V(K) \rightarrow\{0,1, \ldots, d\}$ is said to be a Sperner map if for each $i_{0}, i_{1}, \ldots, i_{k}$ with $0 \leq i_{0}<i_{1}<\ldots<i_{k} \leq d$ and for each $v \in V(K) \cap a_{i_{0}} a_{i_{1}} \ldots a_{i_{k}}, \varphi(v) \in\left\{i_{0}, i_{1}, \ldots, i_{k}\right\}$. A matroid $M$ on $V(K)$ is called a Sperner matroid over $K$ if for each $S \subset\left\{a_{0}, a_{1}, \ldots, a_{d}\right\}$ and for each $v \in V(K) \cap \operatorname{conv}(S), v \in \operatorname{cl}_{M}(S)$, where $\operatorname{conv}(S)$ stands for the convex hull of $S$ and $c l_{M}(S)$ denotes the closure of $S$ in $M$. Let $M$ be a Sperner matroid over $K$ such that $\left\{a_{0}, a_{1}, \ldots, a_{d}\right\}$ forms a basis of $M$. Put

$$
F_{j} \equiv c l_{M}\left(\left\{a_{0}, a_{1}, \ldots, a_{j}\right\}\right)(j=0,1, \ldots, d) .
$$

Let us define $\varphi: V(K) \rightarrow\{0,1, \ldots, d\}$ by setting

$$
\varphi(v) \equiv \begin{cases}0 & \text { if } \quad v \in F_{0}, \\ j & \text { if } \quad v \in F_{j} \backslash F_{j-1}(j=1,2, \ldots, d) .\end{cases}
$$

Received March 20, 2005.

Communicated by Jen-Chih Yao.

2000 Mathematics Subject Classification: 25A99, 52C40, 55N35.

Key words and phrases: Sperner map, Sperner matroid.

Research supported in part by National Science Council of the Republic of China. 
The problem that we consider in this paper is the following: Under what conditions can $\varphi$ become a Sperner map ? It is clearly necessary that the $(d-1)$-face $a_{1} a_{2} \ldots a_{d}$ contains no loops of $M$. To see this, if $a_{1} a_{2} \ldots a_{d}$ contains a loop $v$ of $M$, then $\varphi(v)=0$ and $v \in V(K) \cap a_{1} a_{2} \ldots a_{d}$. As $\varphi$ is a Sperner map, we have $\varphi(v) \in$ $\{1,2, \ldots, d\}$, in contradiction. What is perhaps surprising is that this condition is also sufficient.

We shall establish the following:

Theorem 1. Let $M$ be a Sperner matroid over a triangulation $K$ of a $d$-simplex $a_{0} a_{1} \ldots a_{d}$ such that $\left\{a_{0}, a_{1}, \ldots, a_{d}\right\}$ forms a basis. Then the map $\varphi$ given in (2) is a Sperner map if and only if the $(d-1)$-face $a_{1} a_{2} \ldots a_{d}$ contains no loops of $M$.

To prove Theorem 1, we need the following

Lemma. Let $B$ be a basis of a matroid $M$. Suppose

(a) $S \subset T \subset B$,

(b) $X \subset B, X \cap T=\emptyset$,

(c) $y \in c l_{M}(T) \backslash c l_{M}(S)$.

Then $y \in \operatorname{cl}_{M}(T \cup X) \backslash \operatorname{cl}_{M}(S \cup X)$.

Proof. Suppose $X \neq \emptyset$, and put

$$
X \equiv\left\{x_{1}, x_{2}, \ldots, x_{m}\right\} .
$$

Then $y \notin c l_{M}\left(S \cup\left\{x_{1}\right\}\right)$. To see this, suppose, by contradiction, that $y \in c l_{M}(S \cup$ $\left.\left\{x_{1}\right\}\right)$. Then, by MacLane-Steinitz exchange property, $x_{1} \in \operatorname{cl}_{M}(S \cup\{y\})$. Since $S \subset T$ and $y \in \operatorname{cl}_{M}(T)$, we have

$$
x_{1} \in c l_{M}(S \cup\{y\}) \subset c l_{M}(T),
$$

which contradicts $x_{1} \notin c l_{M}(T)$. Thus we have shown that

(a) ${ }^{\prime} S \cup\left\{x_{1}\right\} \subset T \cup\left\{x_{1}\right\} \subset B$,

(b) $\left\{x_{2}, x_{3}, \ldots, x_{m}\right\} \subset B,\left\{x_{2}, x_{3}, \ldots, x_{m}\right\} \cap\left(T \cup\left\{x_{1}\right\}\right)=\emptyset$,

(c) $)^{\prime} y \in c l_{M}\left(T \cup\left\{x_{1}\right\}\right) \backslash c l_{M}\left(S \cup\left\{x_{1}\right\}\right)$.

The same argument applies (a) $)^{\prime}$, (b) $)^{\prime}$, and (c) $)^{\prime}$ now yielding $y \notin c l_{M}\left(S \cup\left\{x_{1}\right\} \cup\right.$ $\left.\left\{x_{2}\right\}\right)$. Iteration of this procedure $m$ times concludes the proof of the lemma.

We now proceed to prove Theorem 1.

Part " only if " is obvious. To prove part "if", let $v \in V(K) \cap a_{i_{0}} a_{i_{1}} \ldots a_{i_{k}}(0 \leq$ $\left.i_{0}<i_{1}<\ldots<i_{k} \leq d\right)$. Since $M$ is a Sperner matroid, we have 


$$
v \in \operatorname{cl}_{M}\left(\left\{a_{i_{0}}, a_{i_{1}}, \ldots, a_{i_{k}}\right\}\right) .
$$

We want to conclude that $\varphi(v) \in\left\{i_{0}, i_{1}, \ldots, i_{k}\right\}$; it is to split the argument into two cases.

Case 1. $v$ is a loop. Then $a_{i_{0}} a_{i_{1}} \ldots a_{i_{k}}$ contains a loop of $M$. It follows from the hypothesis that $i_{0}=0$. Thus $\varphi(v)=0 \in\left\{i_{0}, i_{1}, \ldots, i_{k}\right\}$.

Case 2. $v$ is not a loop. It follows from (3) and

$$
c l_{M}\left(\left\{a_{i_{0}}\right\}\right) \subsetneq c l_{M}\left(\left\{a_{i_{0}}, a_{i_{1}}\right\}\right) \subsetneq \ldots \subsetneq c l_{M}\left(\left\{a_{i_{0}}, a_{i_{1}}, \ldots, a_{i_{k}}\right\}\right)
$$

that either $v \in c l_{M}\left(\left\{a_{i_{0}}\right\}\right)$ or there exists $p \in\{1,2, \ldots, k\}$ such that

$$
v \in c l_{M}\left(\left\{a_{i_{0}}, a_{i_{1}}, \ldots, a_{i_{p}}\right\}\right) \backslash c l_{M}\left(\left\{a_{i_{0}}, a_{i_{1}}, \ldots, a_{i_{p-1}}\right\}\right) .
$$

If $v \in c l_{M}\left(\left\{a_{i_{0}}\right\}\right)$, then $v \in c l_{M}\left(\left\{a_{0}, a_{1}, \ldots, a_{i_{0}}\right\}\right)$ and, since $v$ is not a loop, $v \notin$ $c l_{M}\left(\left\{a_{0}, a_{1}, \ldots, a_{i_{0}-1}\right\}\right)$ if $i_{0} \geq 1$. Therefore $v_{0} \in F_{0}$ if $i_{0}=0 ; v \in F_{i_{0}} \backslash F_{i_{0}-1}$ if $i_{0} \geq 1$. It follows that $\varphi=i_{0} \in\left\{i_{0}, i_{1}, \ldots, i_{k}\right\}$. Finally, if

$$
v \in c l_{M}\left(\left\{a_{i_{0}}, a_{i_{1}}, \ldots, a_{i_{p}}\right\}\right) \backslash c l_{M}\left(\left\{a_{i_{0}}, a_{i_{1}}, \ldots, a_{i_{p-1}}\right\}\right),
$$

then, by our lemma, we have

$$
v \in c l_{M}\left(\left\{a_{0}, a_{1}, \ldots, a_{i_{p}}\right\}\right) \backslash c l_{M}\left(\left\{a_{0}, a_{1}, \ldots, a_{i_{p-1}}\right\}\right) .
$$

Thus $F_{i_{p-1}} \subsetneq F_{\varphi(v)} \subset F_{i_{p}}$, so that $i_{p-1}<\varphi(v) \leq i_{p}$. We claim that $\varphi(v)=i_{p}$. Suppose $\varphi(v)<i_{p}$. Then we have

$$
v \in c l_{M}\left(\left\{a_{0}, a_{1}, \ldots, a_{i_{\varphi(v)}}\right\}\right) \backslash c l_{M}\left(\left\{a_{i_{0}}, a_{i_{1}}, \ldots, a_{i_{p-1}}\right\}\right),
$$

so that, by our lemma,

$$
v \in \operatorname{cl}_{M}\left(\left\{a_{0}, a_{1}, \ldots, a_{i_{\varphi(v)}}, a_{i_{p}}\right\}\right) \backslash c l_{M}\left(\left\{a_{i_{0}}, a_{i_{1}}, \ldots, a_{i_{p}}\right\}\right),
$$

in contradiction to $v \in c l_{M}\left(\left\{a_{i_{0}}, a_{i_{1}}, \ldots, a_{i_{p}}\right\}\right)$. This contradiction shows that $\varphi(v)=i_{p}$, and we conclude that $\varphi(v) \in\left\{i_{0}, i_{1}, \ldots, i_{k}\right\}$.

This completes the proof.

\section{A Sign FunCtion}

Let $K$ be a triangulation of a $d$-simplex $a_{0} a_{1} \ldots a_{d}$ in a Euclidean space, $M$ a Sperner matroid over $K$, and $B \equiv\left(a_{0}, a_{1}, \ldots, a_{d}\right)$ an ordered basis of $M$. Let $\Lambda_{B}: K \rightarrow\{-1,0,1\}$. We define $\Lambda_{B}\left(v_{0} v_{1} \ldots v_{d}\right)=1$ (resp.-1) if $v_{0} \in F_{0}$ and 
$v_{j} \in F_{j} \backslash F_{j-1}(j=1,2, \ldots, d)$, where $F_{j}(j=0,1, \ldots, d)$ are given in (1), and $\operatorname{det}\left(\alpha_{i j}\right)>0$ (resp. $\left.\operatorname{det}\left(\alpha_{i j}\right)<0\right)$, where

$$
\left(\begin{array}{l}
v_{0} \\
\vdots \\
v_{d}
\end{array}\right)=\left(\begin{array}{ccc}
\alpha_{00} & \cdots & \alpha_{0 d} \\
\vdots & & \vdots \\
\alpha_{d 0} & \cdots & \alpha_{d d}
\end{array}\right)\left(\begin{array}{c}
a_{0} \\
\vdots \\
a_{d}
\end{array}\right), \sum_{j=0}^{d} \alpha_{i j}=1(0 \leq i \leq d) .
$$

We define $\Lambda_{B}\left(v_{0} v_{1} \ldots v_{d}\right)=0$ otherwise. Let $\varphi: V(K) \rightarrow\{0,1, \ldots, d\}$. We call a $d$-simplex $v_{0} v_{1} \ldots v_{d} \in K$ positively (resp. negatively) completely labelled if $\varphi\left(v_{j}\right)=j(j=0,1, \ldots, d)$, and $\operatorname{det}\left(\alpha_{i j}\right)>0\left(\right.$ resp. $\left.\operatorname{det}\left(\alpha_{i j}\right)<0\right)$, where the matrix $\left(\alpha_{i j}\right)$ is given in (4). A $d$-simplex of $K$ is completely labelled if it is positively or negatively completely labelled. It is obvious that $v_{0} v_{1} \ldots v_{d} \in K$ is completely labelled if and only if $\left\{\varphi\left(v_{0}\right), \varphi\left(v_{1}\right), \ldots, \varphi\left(v_{d}\right)\right\}=\{0,1, \ldots, d\}$. The celebrated Sperner lemma [7] asserts that if $\varphi$ is a Sperner map then $\sharp\{\sigma \in K ; \sigma$ is completely labelled $\} \equiv 1(\bmod 2)$. The oriented Sperner lemma [1] states that

$\sharp\{\sigma \in K ; \quad \sigma$ is positively completely labelled $\}-\sharp\{\sigma \in K ; \sigma$ is negatively completely labelled $\}=1$.

By Thoerem 1 and the oriented Sperner lemma, we have

Theorem 2. Let $K$ be a triangulation of a $d$-simplex $a_{0} a_{1} \ldots a_{d}$ in a Euclidean space, and $M$ a Sperner matroid over $K$ such that the $(d-1)$-face $a_{1} a_{2} \ldots a_{d}$ contains no loops of $M$. If $B \equiv\left(a_{0}, a_{1}, \ldots, a_{d}\right)$ is an ordered basis of $M$, then

(a) $\sum_{\sigma \in K} \Lambda_{B}(\sigma)=1$

(b) $\sum_{\sigma \in K}\left|\Lambda_{B}(\sigma)\right| \equiv 1(\bmod 2)$.

Proof. Note that (b) is a consequence of (a). We need only prove (a). By hypothesis, we can retrieve a Sperner map $\varphi$ given in (2). As for each $\sigma \in K$, $\Lambda_{B}(\sigma)=1$ (resp. $\Lambda_{B}(\sigma)=-1$ ) if and only if $\sigma$ is positively (resp. negatively) completely labelled, (a) follows from the oriented Sperner lemma.

Theorem 2 was recently proved by the authors in [4] with a completely different proof. An example given in [4] shows that the condition " the $(d-1)$-face $a_{1} a_{2} \ldots a_{d}$ contains no loops " cannot be dispensed with. Theorem 2(b) is a generalization of Lovász's theorem. It complements Lovász's theorem in two aspects: one concerns an arbitrary matroid while the other is the assertion of oddness. Indeed, Lovász [2] proved the following.

Theorem 3. Let $K$ be a triangulation of a d-simplex $a_{0} a_{1} \ldots a_{d}$ in a Euclidean space, and $M$ a Sperner matroid over $K$ without loops. If $\left\{a_{0}, a_{1}, \ldots, a_{d}\right\}$ is a 
basis of $M$, then $K$ has a d-simplex $v_{0} v_{1} \ldots v_{d}$ such that $\left\{v_{0}, v_{1}, \ldots, v_{d}\right\}$ is also a basis of $M$.

Finally, let us mention that it is perhaps worth developing a general matroid version which contains multiple balanced Sperner lemma [6], combinatorial Lefschetz fixed-point formula [5], and multiple combinatorial Stokes' theorem [3].

\section{REFERENCES}

1. A. B. Brown and S. S. Cairns, Strengthening of Sperner's lemma applied to homology, Proc. Nat. Acad. Sci. USA, 47 (1961), 113-114.

2. L. Lovász, Matroids and Sperner's lemma, Europ. J. Combinatorics, 1 (1980), 65-66.

3. S. N. Lee and M. H. Shih, A counting lemma and multiple combinatorial Stokes' theorem, Europ. J. Combinatorics, 19 (1998), 969-979.

4. S. N. Lee and M. H. Shih, Sperner matroid, Archiv der Mathematik, 81 (2003), 103-112.

5. M. H. Shih and S. N. Lee, A combinatorial Lefschetz fixed-point formula, J. Combinatorial Theory, Ser. A, 61 (1992), 123-129.

6. M. H. Shih and S. N. Lee, Combinatorial formulae for multiple set-valued labellings, Math. Ann., 296 (1993), 35-61.

7. E. Sperner, Neuer Beweis fur die Invarianz der Dimensionszahl und des Gebietes, Abh. Math. Sem. Univ. Hamburg, 6 (1928), 265-272.

Shyh-Nan Lee

Department of Mathemathics,

Chung Yuan Christian University,

Chung Li 320, Taiwan.

E-mail: nan@math.cycu.edu.tw

Mau-Hsiang Shih

Department of Mathematics,

National Taiwan Normal University,

Taipei 116, Taiwan.

E-mail: mhshih@math.ntnu.edu.tw 